\title{
Philosophiques
}

\section{Julien Bigras. Le choc des oeuvres d'art. Montréal, Hurtubise HIMH, 1980, (Collection « Brèches ») 128 p.}

\section{Josiane Boulad Ayoub}

Volume 8, numéro 1, avril 1981

URI : https://id.erudit.org/iderudit/203164ar

DOI : https://doi.org/10.7202/203164ar

Aller au sommaire du numéro

Éditeur(s)

Société de philosophie du Québec

ISSN

0316-2923 (imprimé)

1492-1391 (numérique)

Découvrir la revue

Citer ce compte rendu

Boulad Ayoub, J. (1981). Compte rendu de [Julien Bigras. Le choc des oeuvres

d'art. Montréal, Hurtubise HMH, 1980, (Collection « Brèches ") 128 p.]

Philosophiques, 8(1), 211-214. https://doi.org/10.7202/203164ar d'utilisation que vous pouvez consulter en ligne.

https://apropos.erudit.org/fr/usagers/politique-dutilisation/ 
Julien BIGRAS. Le choc des oeuvres d"art. Montréal, Hurtubise $\mathrm{HMH}$, 1980, (Collection «Brèches») 128 p.

La simple présence d'une oeuvre d'art suffit à provoquer chez chacun de nous un choc global qui se traduit par du plaisir ou du déplaisir. Et si le dilettantisme de l'amateur se satisfait de cet effet pour affirmer son choix et déclarer aimer ou ne pas aimer l'oeuvre, l'esthéticien tente de la comprendre pour la juger. Il ne considère pas une oeuvre d'art exclusivement du point de vue, nécessairement plus limité, de celui qui la regarde mais du point de vue de celui qui l'a faite, de son auteur qui, à son insu ou consciemment, propose dans sa production la solution d'un ou de plusieurs problèmes.

Bouleversé par sa rencontre fortuite avec Bruegel, au musée de Vienne, Julien Bigras nous dit avoir été conduit à repenser ses attitudes esthétiques, et, 
pour commencer, à sonder la "raison" de son plaisir au contact de certaines oeuvres. Mettant à contribution sa formation et sa pratique de psychanalyste, il pose une question radicale: "pourquoi le choc des oeuvres d'art?" Il choisit d'approcher le "mystère" de l'objet esthétique à partir du sujet, entendu doublement: d'une part, l'artiste que cet objet révèle, et, par conséquent, Bigras recherchera la nature des liens entre telle oeuvre et le travail de l'inconscient de son créateur; d'autre part, le sujet est aussi le spectateur qui recrée en quelque sorte l'oeuvre en soi, et l'analyste s'attachera à préciser ce qui se passe dans cet acte de re-création, au cours de ce "coup de foudre» ménagé entre deux personnes par la médiation de l'oeuvre d'art.

Je ferais ici quelques réserves d'ordre surtout méthodologique. Bigras place son investigation dans le cadre d'une étude psychanalytique. Il met au jour des éléments essentiels pour l'étude de la présence de l'artiste dans son oeuvre ou pour interpréter l'envoûtement du contemplateur esthétique par le créateur. Cependant, reste un pas que Bigras franchit aisément, lorsqu'il considère la personnalité de l'artiste ou celui du spectateur comme l'unique voie d'accès à l'oeuvre, voire la clé maîtresse de sa réalité. La lecture qui analyse les fins conscientes de l'artiste, ses idées avouées reste tout aussi nécessaire. La valeur de l'activité artistique ne tient pas seulement à celle qui lui est conférée par l'inconscient et son travail. De plus, ainsi que l'ont montré un Francastel ou un Bourdieu, on ne peut plus négliger de saisir les signes, dans l'objet esthétique, du milieu historique ou social, de «tout cet ensemble de conditions et de déterminations par lequel une oeuvre, toute personnelle qu'elle soit, porte la marque de son époque, son style au sens collectif du terme, d'où se distingueront les styles propres à chaque artiste». Enfin, pour l'esthétique contemporaine, l'étude des lois et nécessités du tableau lui-même a supplanté celle des lois et nécessités des objets représentés dans le tableau. C'est dire que l'intérêt pour les jeux formels de la reproduction distingue actuellement les recherches de l'esthétique de l'art plastique. La «vérité en peinture» s'attacherait non plus tant à ce que le tableau représente qu'à la façon surtout dont il le représente, à la forme. Le signifié serait ici immanent au signifiant.

Ces réserves n'entament en rien, bien entendu, l'intérêt de l'ouvrage ni l'honnêteté du propos. Bigras n'a pas voulu commettre un traité supplémentaire d'esthétique et de méthodologie esthétique. La série des révélations d'une grande oeuvre n'est jamais close, et c'est pourquoi l'art admet la multiplicité sinon le conflit de l'interprétation. À l'exemple de Freud, l'auteur tient à rendre compte de cette part inconsciente dans toute expression consciente ainsi que de sa valeur. Il livre au lecteur, à partir de cette perspective, son exploration du discours artistique et de la relation passionnelle qui réunit quelquefois créateur et spectateur. Et c'est à l'intérieur de ces limites qu'il faudra accepter de le dire; limites qui tiennent à la fois des types d'art pictural étudiés et du parti pris de l'explication. L'auteur a la prudence de ne pas généraliser ses intuitions ou encore de ne pas les appliquer à des oeuvres qui, importantes sur le plan de l'histoire de l'art et de son développement, auraient toutefois manqué de la séduction particulière à d'autres plus célèbres. 
Le choc de Freud devant Michel-Ange, nous fait remarquer Bigras, renvoie non pas au problème posé par le Moïse tel qu'en lui-même l'apparaître le change, mais à la situation familiale de Freud. Celui qui est touché par Moïse est le Freud enfant, en plein désarroi, ne maîtrisant pas sa relation avec les figures du père et de la mère. L'auteur nous avoue que, pour sa part, le Moïse ne lui dit rien. Est-ce à dire qu'il aurait vécu autrement que Freud les schémas possibles de la sexualité infantile? Et si le lecteur d'aventure a été bouleversé par le Moïse, quelle conclusion en tirera-t-il? Et que devient Mö̈se de MichelAnge dans tout cela? Ce qui gêne, dans ce chapitre du livre, c'est que le discours de la psychanalyse tout court l'emporte sur le discours du psychanalyste à propos du choc causé par l'oeuvre d'art. Cette dernière peut-elle être jugée en fonction du degré de fascination qu'elle exerce sur le spectateur? On pourrait mettre en question l'opportunité d'un tel prolégomène subjectif une oeuvre doit me toucher pour en parler - à la critique.

Bigras commente ensuite le travail de Freud sur les tableaux de Léonard de Vinci. Le spectateur, cette fois, est le Freud adulte qui s'identifie, en quelque sorte, à la manière du peintre, sur le plan de la méthode scientifique et du destin de son exposition. De plus, la fascination de Freud devant le sourire ou le regard de Mona Lisa, Saint Jean Baptiste, ou Sainte Anne révèle l'objet du désir de Léonard. La thèse de Bigras est que le Freud charmé par ces oeuvres, c'est l'enfant charmé par sa mère. Le spectateur, dont Freud serait la figure exemplaire, retrouve devant l'oeuvre qui le captive la passivité caractéristique de l'enfant. L'auteur relie les hypothèses de Freud sur la sexualité infantile à celles de son analyse de l'oeuvre de Léonard de Vinci. Le peintre, tout comme quiconque le regarde, redevient l'enfant fasciné par sa mère: le sourire de Mona Lisa est le sourire de sa mère, à Léonard, à Freud, à Bigras, au spectateur, et son charme continue à s'exercer, toujours-déjà. Le «secret» de l'oeuvre d'art, le «secret» du génie de Léonard se découvre au moyen de la psychanalyse du peintre: "Voilà donc le génie de Léonard. Il a touché du doigt, dans ses oeuvres, au secret le plus précieux et le plus intime auquel nous attachons tous le plus grand prix. Voilà également le génie de Freud. Il a lui aussi touché du doigt à ce secret concernant les origines incestueuses de la vie» (p. 71-72). S'agirait-il tout bonnement de comprendre son choc pour comprendre dans sa plénitude le secret du peintre? Ne faudrait-il pas penser au contraire que le secret du peintre est pris, et désormais difficilement dissociable, dans un «secret» qui le déborde, le secret de la peinture elle-même une fois achevée. Il faut peut-être un génie pour retrouver sa mère, comme le dit Bigras, mais ce n'est pas seulement en retrouvant sa mère que nous comprendrons le secret de la formation du génie ou de l'acte créateur.

Si Mö̈se ne touchait guère l'auteur, Bruegel en revanche, l'a assailli. Il a beaucoup à dire sur lui, Bigras, Bruegel. Le thème récurrent chez le peintre de l'enfant seul, ou celui de son massacre ne serait pas entièrement explicable par l'évocation de visées politiques. Bigras préfere interroger le Flamand sur «la souffrance de la séparation entre mère et enfant». Et si Bruegel parle de façon privilégiée à Bigras, c'est qu'il y a correspondance immédiate entre son oeuvre et tous ceux qui s'identifient aux souffrances des petits, des enfants. «Bruegel 
se pose la question de l'enfant perdu, comme Léonard de Vinci, comme Freud, comme moi» (p. 81).

Le commentaire de Freud sur la Gradiva permet à Bigras de consolider ses propositions. Le choc éprouvé au contact de la Gradiva conjure des éléments effrayants: sacrilège, viol, castration. Norbert Hanold a été séduit, tout comme Freud qui écrit ce récit pour séduire Jung et dont la sexualité féminine se manifeste, mais ce texte nous concerne tous par la gravité de ce qu'il met en jeu: la découverte du corps de la mère et de sa réserve de plaisir. Bigras cite, conjointement à la Gradiva, un court écrit de Freud, La tête de Méduse, qui dévoile les positions freudiennes à l'égard du sexe féminin, du sexe de la mère: «Regarder la tête de Méduse, le sexe maternel, pétrifie d'effroi» (p. 119). À la suite du regard sacrilège porté sur le sexe maternel, peut se monter la séquence suivante: castration - pétrification - érection (p. 120). Bigras exige «désormais» que toute théorie sur l'oeuvre d'art fasse sa place à ce mystère: . . « la sexualité féminine - ou plutôt maternelle - tant de l'artiste que de la sexualité féminine de toute mère, et partant de notre propre sexualité infantile, que nous soyons nous-mêmes garçon ou fille» (p. 123). On pourrait peut-être compléter cette exigence et faire place aussi, pourquoi pas, au mystère de la sexualité masculine de l'artiste femme: Madame Vigée-Lebrun par exemple ou Rosa Bonheur?

Bigras conclut en disant que devant certaines oeuvres d'art, comme devant l'image de la mère, il convient de savoir fermer les yeux, dans le silence. Mais entendra-t-on alors les "voix du silence» ou bien celles, fort loquaces, de l'inconscient?

Josiane Boulad Ayoub Université du Québec à Montréal 